\title{
Un campo agonístico con vocación transdisciplinaria: el Desarrollo Humano
}

\author{
Gardy Augusto Bolívar Espinoza* \\ Óscar Cuéllar Saavedra**
}

\begin{abstract}
Resumen: Un nuevo campo de las ciencias sociales aparece masivamente en los noventa mediante el Índice de Desarrollo Humano (IDH) elaborado por el PNUD. Sin embargo, su prehistoria es vasta y ha convocado grandes pensadores desde la antigüedad. Tres nombres aparecen principalmente en los cincuenta, Sen, Arrow y Rawls que inauguran una gran síntesis que reúne, principalmente, la economía, la ética, las ciencias políticas y la filosofía. Posteriormente, no han dejado de proliferar nuevas metodologías e importantes discusiones teóricas en múltiples seminarios internacionales. El artículo se propone exponer las grandes líneas de este campo de estudio. El propósito es afinar los planos en que se desarrolla el campo, y suscitar el interés de desarrollar esta temática como un nuevo paradigma transdisciplinario.
\end{abstract}

Palabras clave: capacidades, opciones igualdad, justicia.

\section{An agnostic field with transdisciplinary vition: Human Development}

\begin{abstract}
A new field of social sciences appears massively in the nineties by means of the Index of Human Development (IDH) elaborated by the PNUD. Nevertheless, its prehistory is vast and has summoned great thinkers from the antiquity. Three names appear mainly in the fifty, Sen, Arrow and Rawls that inaugurate a great synthesis that reunites, mainly, economy, ethics, political sciences and philosophy. Later, new methodologies and important theoretical discussions in multiple international seminaries have proliferate. The article sets out to expose the great lines of this field of study. The intention is to clarify the areas in which this field is developed, and to provoke the interest to develop this thematic as a new transdisciplinarian paradigm.
\end{abstract}

Key words: capacities, options equality, justice.

Recibido: 04.11.2008

Aprobado: 19.12.2008

$* * *$

\footnotetext{
* Universidad Autónoma Metropolitana, Unidad Azcapotzalco, Ciudad de México, México. Email: abe88@hotmail.com

** Universidad Autónoma Metropolitana, Unidad Azcapotzalco, de Ciudad de México, México. Email: oscarcuellar00@prodigy.net.mx.
} 


\section{Introducción}

\section{El problema}

Pese al enorme crecimiento económico alcanzado en las décadas posteriores a la Segunda Guerra, la satisfacción de las necesidades mínimas de la gran mayoría de la población mundial ha continuado siendo insuficiente, desigual e injusta. Este caudal se ha concentrado en forma creciente en exclusivas regiones del mundo; $y$, al interior de casi todos los países del orbe, la desigualdad se ha convertido en norma. Por otra parte, gran parte de esta mayor riqueza se explica por la destrucción de la naturaleza acuciada por el beneficio inmediato de intereses particulares. Los credos y promesas del capitalismo liberal, el socialismo y la social democracia no han impedido la desigualdad y probablemente la han incrementado pese a las propuestas de auto regulación del mercado, la dirección dictatorial de la economía o la de un estado de Bienestar que acumula los defectos de ambos.

\section{La inauguración de un campo}

En los noventa, un nuevo campo de las ciencias sociales, publicitado masivamente por el Índice de Desarrollo Humano (IDH) elaborado por el PNUD, enfrenta directamente el problema de la pobreza y la justicia social y su posible solución. Relevantes instituciones tanto internacionales como nacionales y diversas organizaciones de la sociedad en general acompañan el desafío. Sin embargo, la prehistoria de la temática es amplia y auspiciada por grandes y múltiples pensadores desde la antigüedad. Pero, desde los setenta diversos enfoques interdisciplinarios de las ciencias sociales, principalmente, desde del mundo de la economía, la política, el derecho y la ética, comienzan a enfocarse hacia este campo problemático para iluminar, con nuevas ideas, antiguos problemas. Algunos de los más destacados intelectuales contemporáneos de las ciencias sociales, se reúnen, formal o informalmente, en torno al concepto de Desarrollo Humano. De diversas nacionalidades, tradiciones y escuelas distintas, provenientes de disciplinas múltiples, con experiencias políticas a veces encontradas, el problema se va construyendo como un vasto mosaico o en un campo agonístico por primera vez visto. No ha querido o no ha podido ser disciplina, pero a pesar de todo y pese ser todavía un campo de preparación para la batalla, ha logrado al menos reunirse entorno a un principios común, el de la dignidad humana, antes considerado pura ideología.

\section{Las temáticas}

Este espacio de discusión acción logra sintetizar parte importante de problemas clásicos tratados especialmente por la filosofía convirtiéndolos en preguntas susceptibles de ser investigadas empíricamente a fin de solucionarlos en la práctica mediante la implementación de políticas públicas. En especial, por ejemplo, la obligación de las instituciones públicas 
de preservar este desarrollo; la sustentabilidad del planeta tanto en el cuidado de los recursos como en la seguridad de seguir disfrutando de ellos para las posteriores generaciones; el respeto a los diferentes grupos sociales y a las minorías, en especial la igualdad entre los géneros, pero con todos los grupos sociales que deben ser tratados con dignidad; la potenciación de las capacidades humanas, la cooperación, la equidad, la sustentabilidad y la seguridad social, el reparto eficiente de los recursos, la gobernabilidad, la pobreza, la justicia y la equidad.

\section{La novedad del campo}

Bajo nuevos conceptos, categorías, métodos y técnicas tanto en su vertiente empírica como hermenéutica se han elaborado indicadores que han abierto un vasto campo para la investigación, las políticas públicas y el debate social. Múltiples prácticas de investigación han enriquecido la concepción original aportando nuevos conceptos, métodos y técnicas sugerentes (Griffin, 2001, pág. 26). En varios países se ha convertido como referente teórico, directo o indirecto, en el diseño de políticas públicas sociales y aparece en la acción de variadas instancias sociales, ONG'S. Investigadores de universidades tanto públicas como privadas han implementado centros de investigación y docencia y colaborado con ideas, teorías, experiencias e información que han conformado un espacio cada vez más abarcador y difuso (Ibarra \& Unceta, 2001).

El artículo expone las grandes líneas de este campo de estudios, principalmente en algunos temas teóricos, y la acción de los organismos internacionales respecto de los índices de desarrollo ${ }^{1}$. El propósito es afinar los planos en que se desarrolla el campo, y suscitar el interés para desarrollarlo como un nuevo paradigma transdisciplinario.

\section{Un campo teórico transdisciplinario. Raíces del DH}

Las raíces teóricas del concepto de desarrollo humano se remontan a lo más selecto del pensamiento clásico en la discusión filosófico—ética. El concepto (DH) revive ideas de libertad y la justicia que se presentan en Aristóteles y Platón y en sus versiones posteriores en todas las épocas, y, en especial, las expuestas paradigmáticamente en el siglo XVIII en las temáticas de la economía clásica y de la crítica a la razón práctica. Estas propuestas no sólo significaban una reiteración de los problemas filosóficos del pasado sino una renovación y una síntesis problemática a la luz de

\footnotetext{
${ }^{1}$ Nos hemos basado profusamente a los informes del PNUD y en especial a López-Calva por resaltar el aspecto más institucionalizado del campo del Desarrollo Humano y también, por supuesto, por la calidad de sus escritos. Del mismo modo hemos recurrido antes que una análisis directo de Sen a lo trabajos de Salcedo (Salcedo M., D. 1994; Salcedo, D.,1998). Las demás referencias parecen en la bibliografía.
} 
las exigencias contemporáneas: el ocaso de la edad moderna, el desarrollo y superación del capitalismo industrial. De ahí las nuevas condiciones materiales exigen nuevas técnicas de análisis.

El generalizado optimismo de la Economía política clásica respecto del desarrollo capitalista fue atemperado desde sus inicios por la sospecha de su incapacidad para satisfacer las necesidades de una población en constante crecimiento. Probablemente desde ese entonces surge la necesidad de investigar, sin rubor, la naturaleza de la felicidad como o hacían los antiguos ${ }^{2}$. Además, la temprana crítica a la economía política no dudó de la capacidad del capitalismo en la producción de bienes aunque sustituyó los sentimientos morales como principio explicativo del modo de producción capitalista por la prosaica explotación del trabajo humano. El equilibrio del mercado apareció para la nueva economía el mejor modo de aprovechar la riqueza. El socialismo vaciló entre la imposibilidad del capitalismo y su posible revisión. Desde allí se inaugura una polémica que subsiste $\mathrm{y}$, que en el siglo XX, a nivel político, se expresó como antagonismo entre capitalismo y socialismo o, de un inestable Estado de bienestar. Las posturas extremas se expresaron en un mercado que sería más eficiente en la medida en que no fuera estorbado por la intervención estatal y una centralización dictatorial de la economía.

Por otra parte, la crítica de la razón práctica compitió con la teoría de los sentimientos morales sin llegar a una ruptura, conviviendo el trascendentalismo con el intuicionismo en la explicación de las acciones humanas. Las consideraciones morales pudieron mantenerse sin contradicción respetando el tribunal de la razón y al observador imparcial. Solo en el siglo XX la ruptura se hace explícita entre, por una parte, el utilitarismo y la obligación moral y, por otra, la economía y la ética. El artífice de esta ruptura en el siglo XX es Robinns (cfr. Sen A., On Ethics and Economics, 1987) escisión que se impone hegemónicamente hasta la irrupción de Sen ${ }^{3}$.

A partir de la década de los cincuenta se comienzan a elaborar los parámetros básicos que definen los problemas del desarrollo como crecimiento y el rol de las instituciones en un nuevo terreno de las ciencias sociales: el Desarrollo Humano. Tres nombres aparecen principalmente desde esa época, Sen, Arrow (Arrow, 1951a) (Arrow, 1951b) y Rawls que

\footnotetext{
${ }^{2} \mathrm{Al}$ decir de Malthus “The professed object of Dr, Adam Smith’s inquiry is the nature and causes of the wealth of nations. There another inquiry, however, perhaps still more interesting, wich occasionally mixes whith it, I mean an inquiry into de causes which affect the happiness of nations” Malthus 1798/1986: 303-4 pasaje citado en Bruni, L. y Porta (Bruni \& Porta, 2005) (Ansa Eceiza, 2008).

${ }^{3}$ Ver en esta misma revista en la sección reseñas el artículo «Sen y algunos escritos que concurren a la constitución del campo del Desarrollo Humano». La riqueza y amplitud del aporte bibliográfico de Amartya Sen nos ha obligado a tratar este aspecto aisladamente. Por otra parte, al constituirse este autor, en el vértice ideológico del nuevo campo a pesar de los infinitos aportes que han hecho otros autores, preferirnos presentar a Sen en forma separada.
} 
comienzan a formar una gran síntesis que reúne la economía, la ética y las ciencias políticas y la filosofía.

A partir de la idea de una economía sostenible, surgen varias formas de definirla: "ecodesarrollo" (Sach, 1981), "crecimiento intensivo" (Rizhkow, 1986), “crecimiento orgánico” (Mesarovic Pestel, 1972), conceptos que se hacen públicos en el Informe Brundtland en 1987 (Bermejo, 2001). Por otra parte, la sicología, las ciencias de la educción, etc., se preocuparon, desde fines de los ochenta, y auspiciados por UNESCO, por el desarrollo humano en un sentido amplio: sicológico, fisiológico, emocional, cultural, etc. ${ }^{4}$ (Ibarra \& Unceta, 2001; Bermejo, 2001).

Este amplio espacio discursivo, en los noventa, tiene por centro la libertad individual que supone opciones y capacidades en un marco de obligación ética institucional. "El objetivo del desarrollo se relaciona con la valuación de las libertades reales que disfruta la gente en una población determinada...La discusión sobre medios y fines del desarrollo nos llama a colocar la perspectiva de la libertad en el centro del escenario. Las personas deben ser vistas, bajo este enfoque, como agentes activamente involucrados — dada la oportunidad — en la construcción de su propio destino y no solamente como receptores pasivos del fruto de ingeniosos programas de desarrollo”. (Sen A., 1999).

\section{Constructores principales}

Entre los múltiples reconocidos investigadores y centros de investigación que han participado en la elaboración e impulso de la teoría del desarrollo humano, destacan como los más próximos fundadores del campo del desarrollo humano Amartya Kumar Sen economista bengalí (1933) y Mahbub ul Haq, paquistaní (1934-1998). El primero afirmaba que el propósito básico del desarrollo humano era ampliar las opciones de la gente, las que pueden ser infinitas, y cambiar con el tiempo. Sostenía que la gente a menudo evalúa los logros, que no se muestran totalmente o no inmediatamente, en figuras de la renta o del crecimiento: mayor acceso al conocimiento, una nutrición y servicios médicos mejores, sustentos más seguros, seguridad contra crimen y violencia física, horas de satisfacción del ocio, libertades políticas y culturales y sentido de la participación en actividades de la comunidad y, en definitiva que el objetivo del desarrollo era crear un ambiente que permitiera a la gente gozar de largas, sanas y creativas vi-

\footnotetext{
${ }^{4}$ Le Centre International de Recherches et Études Transdisciplinaires (CIRET) asociación fundada en 1987 se propuso desarrollar la investigación científica desde una nueva perspectiva cultural: la transdisciplinariedad. Su objetivo considera el flujo de información que circula de una disciplina a otra para crear un lugar privilegiado de encuentro y diálogo entre especialistas de diferentes ciencias y de otros dominios, particularmente los especialistas de la educación. El objetivo del centro se precisa en su proyecto moral vinculado a la UNESCO. (CIRET-UNESCO, 10 Février 1997)
} 
das $^{5}$. Por su parte Sen, en el alba del siglo XXI, ponía el acento en la libertad, principio de los logros materiales.

Mahbub ul Haq fue el coordinador de los primeros informes del PNUD, trabajó como director de Planificación en el Banco Mundial (19701982), ministro de Planificación y Finanzas (1982-1984) fundador de Human Development Centre (Centro para el Desarrollo Humano), en Pakistán, en 1996.

También participa en forma significativa Gro Harlem Brundtland política noruega (1939), doctora en medicina y master en Salud Pública, miembro del Partido Laborista noruego, ministra de Medio Ambiente en 1974 y Primera Ministra de Noruega en varios periodos; directora general de la Organización Mundial de la Salud (OMS) de 1998 a 2003, es autora del famoso informe que lleva su nombre (Brundtland, 1987). Ella ha sostenido que la violencia era el principal problema de salud pública.

\section{Bienestar y capacidades}

Aunque el concepto de capacidades está relacionado con el tema de bienestar en general, su tratamiento contemporáneo se originó en las conferencias de Sen, en la Universidad de Stanford en 1979, enfocadas a analizar distintas interpretaciones del punto de vista igualitario. Con una crítica al utilitarismo y al rawlsianismo. Sen desarrolló la idea de funcionamiento y propuso las capacidades como respuesta alternativa a la pregunta: ¿desigualdad, de qué? (Sen A., Equality of What?, 1980). En una serie de artículos subsecuentes, estableció las capacidades como un enfoque general para evaluar la condición de bienestar individual (cfr., Sen A., Commodities and Capabilities, 1985; PNUD, Estudios sobre el desarrollo humano, Num. 2006-6, 2003-6).

Sen empieza con una evaluación del utilitarismo como principio moral. El utilitarismo requiere que, dada una elección entre varias alternativas, se seleccione la que maximiza la suma total de utilidad entre todos los seres humanos, teniendo como referencia clásica la perspectiva de utilitarismo de Bentham (1789) (Bentham, 1789) cuyos principios axiomáticos han sido desarrollados posteriormente por (D’Apremont \& Gevers, 1977) (Maskin, 1978) (Roberts, 1980) (Basu, 1983). Esta idea, combinada con el supuesto estándar de que la utilidad marginal de cada persona disminuye

\footnotetext{
5 "The basic purpose of development is to enlarge people's choices. In principle, these choices can be infinite and can change over time. People often value achievements that do not show up at all, or not immediately, in income or growth figures: greater access to knowledge, better nutrition and health services, more secure livelihoods, security against crime and physical violence, satisfying leisure hours, political and cultural freedoms and sense of participation in community activities. The objective of development is to create an enabling environment for people to enjoy long, healthy and creative lives."
} 
conforme su ingreso aumenta, implica, para el utilitarismo, que cuando un ingreso fijo se distribuye entre un conjunto de individuos, debería hacerse de forma tal que cada persona obtenga la misma utilidad marginal. De ahí que a la pregunta “¿igualdad, de qué?”, la respuesta utilitarista sea: utilidad marginal (PNUD, Estudios sobre el desarrollo humano, Num. 2006-6, 2003-6).

Sen argumenta entonces que lo anterior es válido cuando los individuos en cuestión son homogéneos, pero no es sostenible cuando reconocemos la diversidad esencial de los seres humanos.

En el desarrollo de las nociones de funcionamiento y capacidad, es un referente obligado el trabajo del filósofo John Rawls. Según él, entre los funcionamientos básicos, que deben proveerse en igualdad a los individuos, se encuentran ciertos "bienes primarios" que incluyen no solamente aspectos materiales, sino aspectos institucionales, de participación política y de realización social ${ }^{6}$. El conjunto de los “funcionamientos", entendidos como las opciones reales disponibles para un individuo, se define como “capacidades”.

La función de conversión, por ejemplo, introduce una gran heterogeneidad entre individuos, heterogeneidad que se consideraba inexistente en los enfoques utilitaristas o bien derivaba en implicaciones inaceptables. Un ejemplo clásico de implicación inaceptable de la regla utilitarista de bienestar social sería la de dar menos bienes a un individuo con alguna discapacidad debido a su imposibilidad de "convertir" dichos bienes en niveles altos de bienestar.

Pensemos en un individuo que tiene acceso a un automóvil. De acuerdo con la visión propuesta, dicho automóvil no proporciona bienestar per se, sino que abre un conjunto de "funcionamientos" disponibles para dicho individuo.

El acceso a ese bien le dota de un conjunto más amplio de capacidades: socializar, trabajar, disfrutar de momentos de recreación familiar, quizás participar en eventos políticos que, de otra manera, no le serían asequibles, mejorar su reputación social y, en consecuencia, su autoestima y su relación con la comunidad. El individuo asignará un valor a cada uno de esos funcionamientos disponibles y evaluará la importancia de dicho conjunto de capacidades para su bienestar. La relación utilitarista que va directamente de mayor acceso a bienes a mayor nivel de utilidad, se ve sustituida por una relación en la que el acceso a bienes abre un conjunto de capacidades, y son éstas las que impactan sobre el bienestar. Ampliar dicho conjunto de opciones reales a disposición de los individuos, visualizando el acceso a bienes y servicios sólo como una parte del proceso, debería ser, según este enfoque, el objetivo último del desarrollo.

\footnotetext{
${ }^{6}$ Una revisión detallada del enfoque de las capacidades, su origen filosófico y las críticas a enfoques alternativos puede encontrarse en Basu y López-Calva (2003).
} 
"Es importante hacer notar que cuando se alude a la ampliación del conjunto de opciones reales, se hace referencia no solamente a las opciones disponibles sino también a las condiciones económicas, institucionales, cognitivas y sociales con las que cuenta el individuo para ejercer su elección. Es en este sentido que se puede definir el desarrollo como una ampliación de la capacidad de elección de los individuos o, en términos filosóficos, como una ampliación de la libertad en el sentido positivo. (LópezCalva \& Vélez Grajales, 2003:1)

Para Berlín cuando no existe un impedimento legal para ejercer cierta acción el individuo es "libre" en el sentido negativo (es decir, no le es negada la opción desde el punto de vista legal o normativo). Sin embargo, el individuo solamente es libre en el sentido positivo cuando es, además, plenamente capaz de ejercer su elección (Berlin, Four Essays on Liberty, 1969) (Berlin, Two Concepts of Liberty, 1969a). La visión de libertad en el sentido negativo es común en los liberales clásicos y en la visión de algunos economistas contemporáneos (ver, por ejemplo, Friedman \& Friedman, 1990). (PNUD, 2003-1, pág. 5)” (ídem).

\section{Desigualdad y justicia: Sen y Rawls}

Con Sen se retoma el tema de la igualdad económica planteando una pregunta central: ¿cuál es la dimensión relevante para medir la desigualdad? Se trata de medir el acceso a bienes y servicios, su "conversión” en planes reales de vida y su evaluación por la satisfacción individual. El argumento central de estos trabajos se basa en la idea de que la medición del bienestar no debe derivarse de indicadores "ex-post”, como lo planteaban los filósofos utilitaristas clásicos al otorgar una importancia central al acceso a bienes y servicios. Estas ideas críticas ya se encuentran en Dobb quien abre un campo problemático al abandonar las teorías del equilibrio general proponiendo que la coordinación ex ante es ampliamente superior a la coordinación ex post que realiza el mecanismo del mercado.

Esta visión utilitarista carece de relevancia normativa según Sen. La nueva propuesta hace énfasis en el carácter instrumental del acceso a bienes y servicios, concibiéndolos únicamente como un medio para poder alcanzar un plan de vida o una realización individual plena. Así, la medición del bienestar debería verse como un proceso con varios componentes: el acceso a bienes y servicios, una función de “conversión” de estos bienes y servicios en opciones reales de planes de vida y, por último, una función de "evaluación" que transforma la elección hecha en un nivel de satisfacción individual (PNUD México, 2003-1). (López-Calva \& Vélez Grajales, 2003-1)

Sen también considera la crítica de Rawls referente al velo de ignorancia detrás del cual, la gente no optaría por una sociedad que maximiza la suma total de utilidad, sino que se inclinaría por una distribución más equitativa. Este autor presenta la injusticia del utilitarismo con algunas de 
nuestras nociones básicas de libertad e igualdad (PNUD, Estudios sobre el desarrollo humano, Num. 2006-6, 2003-6). (López-Calva L. F., 2003-6)

Rawls propuso un principio según el cual la sociedad es evaluada en términos del nivel alcanzado por la persona que está en una situación peor dentro de esa sociedad, medido sobre un índice de bienes primarios -el llamado principio maximin- $-\S$ Los economistas comúnmente le atribuyen un principio que sigue el mismo criterio, pero que mide el nivel sobre la dimensión de la utilidad. (PNUD, Estudios sobre el desarrollo humano, Num. 2006-6, 2003-6) (López-Calva L. F., 2003-6)

Rawls (1971), sin embargo, se alejó de la definición tradicional de bienestar, eliminó el énfasis en la utilidad y propuso una visión basada en lo que llamó bienes primarios. El criterio de Rawls sería el primer paso hacia una teoría formal en que la igualdad de oportunidades se vuelve el concepto de importancia moral para la justicia distributiva. El principio rawlsiano de justicia puede ser resumido en el siguiente mandato: maximiza el mínimo, sobre todas las personas, del acceso al conjunto de bienes primarios. Este mandato es llamado el principio de diferencia. La definición de bienes primarios es, por lo tanto, esencial para el entendimiento de la teoría de Rawls. Con base en Rawls, podemos identificar cinco grupos de bienes primarios (ídem):

a) Libertades básicas,

b) Libertad de movimiento y elección de ocupación,

c) Capacidad de acceso a posiciones de responsabilidad pública,

d) Ingreso y riqueza,

e) Las bases sociales del auto-respeto

Es importante señalar el argumento de Sen, en el sentido de que el principio de diferencia de Rawls puede ser criticado por estar enfocado a medios (bienes) y no a fines (libertades). Así, se le puede atribuir una tendencia fetichista hacia bienes primarios en analogía con la discusión de Marx (1844) de fetichismo hacia los bienes

Retomando el velo de ignorancia como argumento que clama que los individuos escogerían el principio maximin sobre un índice de bienes primarios como el contrato social aceptable, según la teoría de Rawls, es interesante abordar el análisis de su formalización. (PNUD, Estudios sobre el desarrollo humano, Num. 2006-6, 2003-6) (López-Calva L. F., 2003-6)

El principio de diferencia defendido por Rawls ha sido criticado por Sen $(1980,1993)$ desde dos perspectivas: primero, reclamando su imparcialidad (justicia como justicia) y segundo, apelando a una ventaja mutua (escogiendo el contrato social detrás del velo de ignorancia). (ídem)

Sin embargo, este principio contribuye a la formación de la noción básica de justicia como igualdad de oportunidades ex-ante, opuesto al énfasis en resultados de la teoría tradicional. Uno de los primeros esfuerzos 
de formalización en este sentido fue hecho por Sen (1985), quien trató de dar estructura a la perspectiva de bienestar basado en los conceptos de funcionamientos y capacidades (PNUD, Estudios sobre el desarrollo humano, Num. 2006-6, 2003-6). (López-Calva L. F., 2003-6)

\section{Índice y teoría}

La discusión que llevó al desarrollo del Índice de Desarrollo Humano (IDH) tiene sus orígenes en la literatura sobre economía del bienestar. Por mucho tiempo la economía del bienestar tradicional estuvo inclinada a identificar el bienestar de una persona con su posesión de bienes y servicios. Esta tendencia derivó en un enfoque basado en el ingreso, ya que, a través de esta variable, se determina cuánto puede consumir un individuo. Este enfoque considera, comúnmente, a cada persona dotada de un cierto ingreso y 'utilidad o función de bienestar', por lo que el consumo es la variable que establece el nivel de utilidad que alcanzado por el individuo. El bienestar social, de acuerdo con este enfoque, se representa agregando los niveles de utilidad de todos los individuos en la sociedad

Una ruta alternativa ha sido el enfoque de capacidades, que reemplaza la importancia tradicional de bienes y utilidad con una visión centrada en funcionamientos y capacidades. Este enfoque de capacidades tiene sus orígenes en el pensamiento de John Stuart Mill y Adam Smith e inclusive se remonta a Aristóteles. En las últimas dos décadas, —a partir de que estos pensamientos teóricos fueron resucitados, reinterpretados y parcialmente formalizados por Amartya Sen $(1980,1985)$ - el enfoque de las capacidades ganó cierto reconocimiento dentro de la profesión económica.

El enfoque de capacidades cuenta ya con una literatura sustancial, pero un punto de discordia en economía del bienestar tradicional se encuentra todavía en la distinción entre bienes y funcionamientos, así como entre logro y libertad. Un funcionamiento es lo que una persona logra ser o hacer. Un bien puede permitir un funcionamiento, pero es distinto a él: una bicicleta es un bien, mientras que poderse transportar rápidamente es un funcionamiento. Dos personas, cada una con una bicicleta, pueden alcanzar funcionamientos diferentes; de hecho, aún con la posesión de la bicicleta, a uno de estos dos individuos podría resultarle imposible alcanzar cierto funcionamiento. Por ejemplo, si una de ellas está discapacitada o no sabe usar la bicicleta, no le sería posible utilizarla para transportarse. Ésta, es una de las distinciones operacionales centrales entre bienes y funcionamientos (ídem)

Así como un funcionamiento no debe ser confundido con un bien, tampoco debe serlo con la utilidad que éste proporciona. De hecho, el enfoque de capacidades no depende del concepto de utilidad. Si se usara la idea de utilidad junto con la de funcionamientos, entonces debería establecerse que los primeros son anteriores a la utilidad. Así como los bienes hacen posible lograr ciertos funcionamientos, éstos a su vez pueden permitir a 
una persona alcanzar ciertos niveles de utilidad. Sin embargo, los funcionamientos logrados por una persona no pueden ser suficientes para determinar la calidad general de vida o el bienestar de una persona. Para esto necesitamos conocer, como mínimo, la capacidad de un individuo, es decir, los funcionamientos entre los cuales pudo elegir y qué pudo haber logrado. Por lo tanto, las capacidades, es decir, el conjunto de funcionamientos disponibles para un individuo, están estrechamente relacionadas con la idea de oportunidad y libertad. De acuerdo con el enfoque de capacidades, al determinar la calidad general de vida de una persona, no es suficiente saber qué funcionamientos alcanzó, también es necesario conocer entre cuáles de éstos eligió (ídem)

Como Sen (Sen A., Development as Freedom, 1999) ha enfatizado, otro aspecto evidente es que, en realidad, la sociedad está formada por individuos cuya calidad de vida no puede ser completamente capturada por un número real, puesto que se habla de caracteres con distintas nociones de bienestar, felicidad, deseo y satisfacción. Lo anterior tiene como consecuencia que la idea de las capacidades no está completamente formalizada y, tal vez, ni siquiera sea completamente “formalizable”. (PNUD, Estudios sobre el desarrollo humano, Núm. 2006-6, 2003-6). (López-Calva L. F., 2003-6)

Entre los economistas existe una tendencia a relacionar la facilidad para formalizar un concepto con el grado de validez del mismo. Sin embargo, existen ideas importantes como, por ejemplo, utilidad, libertad o felicidad que pueden ser imposibles de capturar completamente en una sola formalización. Eso no reduce, de ninguna manera, su relevancia conceptual. Una consecuencia de adoptar tal punto de vista es que, al tratar de comparar empíricamente la calidad de vida lograda por diferentes sociedades a través del enfoque de capacidades, es necesario enfocarse en unos cuantos funcionamientos. Así, surgen las preguntas: saludable?

¿Tiene la gente en una sociedad X la opción de una vida larga $y$

¿Está preparada para leer, escribir y, por lo tanto, acceder a la información, desenvolverse en su medio, disfrutar de la literatura y la comunicación con otros? ¿tiene suficientes recursos para comer y beber adecuadamente? (PNUD, Estudios sobre el desarrollo humano, Num. 2006-6, 20036) (López-Calva L. F., 2003-6).

\section{Los orígenes del Î́ndice de Desarrollo Humano PNUD}

El tema Desarrollo humano y de la justicia ha alcanzado especial relevancia desde la segunda guerra sobretodo en los años noventa y, fundamentalmente, desde principios del nuevo siglo. Concebido a fines de los años ochenta, en los noventa, alcanza su institucionalización al interior de 
las ciencias sociales y en la política efectiva. Esta presencia se manifiesta, entre otras razones, por el liderazgo e impulso de organismos internacionales ligados a las Naciones Unidas, BID, CEPAL, OECD y a la OIT.

En especial, el PNUD se ha dedicado a promover una serie de iniciativas en cuyo centro se encuentra el concepto de Desarrollo Humano. El Programa de las Naciones Unidas para el Desarrollo (PNUD) ha promovido el concepto de desarrollo humano definido como el proceso de ampliación de las posibilidades de elegir de los individuos que tiene como objetivo expandir la gama de oportunidades abiertas a las personas para vivir una vida saludable, creativa y con los medios adecuados para desenvolverse en su entorno social ${ }^{7}$.

Desde el año 1990 ha elaborado Informes Internacionales anuales, a los cuales se han sumado más de ochenta; edita una Revista Latinoamericana de desarrollo humano así como un Boletín mensual de desarrollo humano que recoge avances de investigación y reflexiones teóricas. Posee 135 sedes en los países de todo el mundo y 166 donde trabaja; ha registrado 5.000 millones de dólares en ingresos totales en 2007; cuenta con 5.593 voluntarios de las Naciones Unidas en todo el mundo; 599 Informes Nacionales han sido emitidos en 135 países; 46 países de los más pobres reciben apoyo del PNUD para competir en el comercio internacional y obtener sus beneficios; 26 países recibieron apoyo electoral en 2007; 122 países ejecutan programas apoyados por el PNUD en materia de energía y medio ambiente (PNUD, mayo 2008).

\section{El índice de desarrollo humano}

El Índice de Desarrollo Humano (IDH) ha sido uno de los resultados más sobresalientes y publicitados en este amplio proceso. Ideado por Mahbub ul Haq y Amartya Sen, es usado desde 1990 por el PNUD (Programa de las Naciones Unidas para el Desarrollo) en su informe anual y se ha convertido en el indicador estándar para los estudios de desarrollo y bienestar entre países. Reemplazó con éxito a los índices de PB/per cápita y al Índice de Calidad de Vida Física inventado por M. D. Morris en los años sesenta (Morris, 1979) (Ibarra \& Unceta, 2001, pág. 30). A éste le han seguido varios índices que pretenden mejorar sus limitaciones o ampliar sus horizontes.

La medición del desarrollo humano es un elemento fundamental para el diseño de las políticas públicas de un país. Entre otros factores, permite

\footnotetext{
${ }^{7}$ En México, El Consejo Nacional de Población (Conapo, 2001) retoma este concepto definiendo al desarrollo humano como un proceso continuo de ampliación de las capacidades y de las opciones de las personas para que puedan llevar a cabo el proyecto de vida que, por distintas razones, valoran. Este concepto enfatiza la noción de que el desarrollo no se explica únicamente con el ingreso de la población y que esta dimensión no representa la suma total de una vida humana (PNUD, 2003-1, pág. 5). (López-Calva \& Vélez Grajales, 2003-1)
} 
evaluar los avances o retrocesos en las condiciones de vida de sus habitantes, establecer la magnitud del problema del desarrollo, caracterizar el fenómeno para el diseño de políticas, programas y acciones del sector público y definir claramente los objetivos que se persiguen en términos de bienestar. Existen en el mundo diversas maneras de medir el desarrollo humano. El indicador más comúnmente utilizado es el Producto Interno Bruto (PIB), que cuantifica la capacidad de una economía para generar satisfactores para su población; sin embargo, este indicador tiene sus limitaciones para reflejar el desarrollo en un sentido más amplio. Precisamente para hacer frente a estas limitaciones, se han desarrollado una serie de alternativas en las que se incluye información adicional sobre múltiples facetas del desarrollo y, en particular, indicadores relacionados con las condiciones sociales de la población.

En la literatura sobre medición del desarrollo se toman en cuenta dos decisiones básicas que determinan finalmente la metodología a utilizar: la(s) dimensión(es) del desarrollo a considerar, y la agregación (ponderación) de los distintos elementos — en caso de existir más de una dimensión. Dichas decisiones, generalmente, se toman en función del objetivo que se persigue en el ejercicio de medición y tienen consecuencias para el diseño de política, ya que sus resultados pueden dirigir las acciones públicas hacia variables o resultados determinados. Por ejemplo, si se decide tomar como medida de desarrollo el producto por habitante o el ingreso per cápita, el sector público puede privilegiar acciones totalmente distintas a las que resultarían en caso de tomar a la diversidad cultural como punto de referencia para evaluar el progreso de una nación (ídem).

De los esfuerzos realizados en el mundo para medir de manera sistemática el desarrollo humano, quizás el más destacado sea el Índice de Desarrollo Humano (IDH), propuesto por el Programa de Naciones Unidas para el Desarrollo (PNUD). Dicho índice combina tres elementos para evaluar el progreso de los países en materia de desarrollo humano: el Producto Interno Bruto (PIB) por habitante, la salud y la educación; cada uno se incluye con la misma ponderación. Debido a su simplicidad y a requisición de información, generalmente disponible para su construcción, se ha convertido en el punto de referencia más utilizado para realizar comparaciones internacionales e incluso muchos países han adoptado los índices de desarrollo humano como instrumento de política y como indicador del éxito o fracaso de sus políticas nacionales (PNUD, Estudios sobre el desarrollo humano, Num. 2006-6, 2003-6). (López-Calva L. F., 2003-6)

\section{Cuantificación e indicadores}

Los indicadores referidos al desarrollo humano (IDH) pretenden una forma "científica" de comprender y explicar problemas antes tratados filosóficamente o teóricamente. La atención a problemas éticos de justicia, libertad y equidad manifiestan una innegable continuidad con los temas filosóficos del siglo XVIII. En este campo en formación proliferan 
sofisticados instrumentos cuantitativos que se aplican a los más diversos temas y niveles del acontecer social; tiene raíces profundas y prestigiadas en la tradición empirista y en un vasto campo sostenido por un individualismo metodológico de orígenes clásicos. Ambas tradiciones han tenido éxitos innegables así como profundas limitaciones. Por otra parte, se han repuesto temas éticos hasta hace poco por concepciones más técnicas. Así, es habitual la reposición ampliamente aceptada que ha propiciado que aparezcan en las acciones de gobierno iniciativas que tienen en la mira la consumación de un "buen gobierno", la equidad, la justicia y la felicidad, para nombrar sólo unos pocos de un amplio espectro. También el cuestionamiento o discusión de los procedimientos técnicos, los supuestos ideológicos que entrañan y la utilidad de las políticas que prohíja son temas de reflexión permanentes. La debilidad de los indicadores e índices de una variable que crece y se complica constantemente, hace perder la noción de qué es lo que se está midiendo. Los diversos niveles sociales a los cuales se aplica indiscriminadamente: individuos, familias, redes, sociedades enteras y múltiples referentes teóricos de desarrollo global.

Emparentados con este referente las “capacidades” han intentado ser medidas desde diferentes perspectivas y se han transformado en parámetros directos o indirectos de las políticas públicas. Pero se mantiene sin crítica y, alternativamente, se reconocen algunos aspectos de la tradición que le dio origen y otras veces los deja de lado sin explicación.

\section{La medición del desarrollo humano a través del IDH: “ingresos" y "capacidades" 8}

Aunque la implementación empírica del enfoque de las capacidades no es una tarea fácil, el índice utilizado internacionalmente incorpora tres funcionamientos básicos: educación, salud y acceso a bienes y servicios mediante el ingreso. Existen variaciones de dicho índice, por ejemplo, las derivadas de corregir por equidad y por potenciación de género. De esta manera se incorporan a su cálculo otras dimensiones no incluidas en la medida convencional

Para medir el desarrollo humano se construyen índices que corresponden a la idea de que el subdesarrollo es una carencia de capacidades básicas más que una carencia de ingreso per se. La introducción del enfoque de las capacidades para sustituir el enfoque del ingreso se ha tratado de reflejar no sólo a nivel conceptual sino en la implementación de medidas multidimensionales del desarrollo. Aunque la evidencia empírica muestra en general una relación fuerte entre ingresos bajos y carencia de capacidades, existen casos en que esta relación no se cumple a nivel poblacional, como se ha mostrado en el caso de los países y las entidades mexicanas, o incluso a nivel familiar e individual. Ravallion (1997) concuerda con el

${ }^{8}$ Cfr., López-Calva \& Vélez Grajales, 2003-1 
PNUD en cuanto a que no hay garantía de que el crecimiento económico beneficie a personas que no tienen acceso a los mercados. En realidad, la falta de conexión con mercados, o la exclusión de los mismos, es una de las razones que impiden que los beneficios del crecimiento se reflejen en ampliación de capacidades. La utilización del índice de desarrollo humano como indicador del nivel de desarrollo de una población supone que el crecimiento y el desarrollo son conceptos relacionados pero son distintos: los indicadores del producto per cápita utilizados por muchos años como medidas del desarrollo de los países, son incompletos y no proporcionan toda la información deseada. Puede suceder que la evaluación de países sea negativa si se considerara sólo su ingreso per cápita y, positiva si esta cambia cuando se agregan otros indicadores y viceversa. (ídem, 1, pág. 12)

Varias preguntas relevantes surgen cuando se intenta establecer una medición multidimensional del desarrollo. Entre las más importantes desde el punto de vista conceptual se encuentran las siguientes: 1 . ¿Cuáles son los funcionamientos básicos a considerar? 2. ¿Cuáles deben ser las ponderaciones adecuadas para agregar las distintas dimensiones? Estas preguntas han sido centrales en las discusiones académicas y todas las propuestas de indicadores multidimensionales se enfrentan a ellas. El PNUD ha utilizado para el cálculo del IDH tres dimensiones básicas: ingreso, educación y salud. Cada dimensión es medida a través de un índice que considera las desviaciones o "rezagos" con respecto a algún valor de referencia, que en general es el logro deseable. Una vez obtenidos los índices en cada dimensión, éstos se agregan con ponderaciones iguales.

\section{Críticas al IDH: Ravallion, Kelley y Srinivasan}

Existen observaciones críticas al índice de desarrollo humano en dos niveles: el conceptual y el de implementación. Las primeras se basan en la discusión sobre las dimensiones relevantes a considerar y su forma de agregación. Ravallion y Kelley señalan que la forma de agregación del IDH por medio de un promedio simple hace posible "un nivel perfecto de sustitución entre sus tres componentes” lo que supone una falta de especificación y énfasis teórico por lo que los juicios detrás de la valoración de las distintas dimensiones del IDH no son explícitos y, por lo tanto, son cuestionables (Ravallion, 1997; (PNUD, 2003-1, págs. 11-14) (López-Calva \& Vélez Grajales, 2003-1)

Kelley (1991), por su parte, observa que el PNUD no presenta un análisis detallado que justifique el uso de la fórmula de agregación del IDH; él sugiere que se dé un peso mayor al PIB per cápita, ya que el ingreso refleja la capacidad de los individuos de escoger entre muchas otras dimensiones del desarrollo humano. Sin embargo, aceptar esta propuesta es acercarse de nuevo al enfoque del ingreso y alejarse del enfoque de las capacidades. Este último autor somete a discusión algunos otros problemas teóricos de la medición del IDH. En primer término, critica la definición de las cotas basadas en valores "ideales" de las variables consideradas, que se 
utilizan para construir los índices de cada componente. Para ilustrar el punto cambia el valor superior o "cota deseada” de la esperanza de vida de 78 a 73 años y encuentra que 22 países pasarían de la categoría de "desarrollo humano bajo" a la de "desarrollo humano medio” y que otros 10 países pasarían de "desarrollo humano medio" a "desarrollo humano alto" 9 . Por último, Kelley (1991) hace notar que la metodología de medición del IDH no permite reflejar el mejoramiento de los países catalogados como desarrollados porque su índice presenta valores que van de 0.96 a 1 (ídem).

Srinivasan (1994) alude a dos cuestiones en lo que al marco conceptual se refiere. La primera tiene que ver con el origen del concepto. En su opinión, el uso del enfoque “alternativo” data desde 1938, año en el que el Indian National Planning Commitee enfatizó la necesidad de lograr mejores niveles de vida para la población a través de la reducción de la pobreza y de la búsqueda de una distribución más equitativa de la riqueza. Así, Srinivasan (1994) defiende la idea de que el rol instrumental del ingreso ha estado presente desde hace tiempo en el pensamiento de los economistas y sobre todo de los planeadores de política pública. Por otra parte, este autor afirma que no hay correspondencia entre el concepto de las capacidades y su implementación a través del IDH debido a que las capacidades no tienen una métrica homogénea, a diferencia del enfoque del ingreso que sí tiene esa característica. Srinivasan señala en este sentido que, dado que el valor relativo de las capacidades varía entre individuos, grupos sociales y países $\mathrm{y}$, tomando en cuenta que los componentes del IDH son funcionamientos en el sentido de Sen, el valor intrínseco de cada funcionamiento no se captura en la medición lineal del IDH. Respecto a la implementación del cálculo del IDH, el autor en cuestión observa que pueden surgir ciertos problemas derivados de la misma construcción de las bases de datos que se utilizan para hacer los cálculos del índice ${ }^{10}$. Además de los posibles sesgos que lo anterior puede generar, señala que el IDH tiene serios problemas para ser comparable en el tiempo y en el espacio principalmente debido a las variaciones que ha sufrido su metodología de cálculo, lo que dificulta las inferencias acerca de los procesos de desarrollo. Si bien las críticas anteriores tienen su grado de validez, es cierto también que la importancia y la utilidad del IDH han sido validadas empíricamente. Hoy en día, los sectores académicos y de política pública aceptan que el IDH provee información relevante sobre el estado del desarrollo desde una perspectiva am-

\footnotetext{
${ }^{9}$ El PNUD clasifica a los países con índices mayores a 0.8 como de "desarrollo humano alto”, a los países con un índice entre 0.5 y 0.799 como de “desarrollo humano medio”, y a los países con índices menores a 0.5 como de "desarrollo humano bajo".

${ }^{10}$ Entre las debilidades que encuentra en las bases de datos están los procedimientos de extrapolación de datos de pocos países a todos los demás para poder construir una medida del PIB homogénea a través del poder de paridad de compra (PPC). Otro problema es que para algunos países menos desarrollados no existe el indicador de esperanza de vida y se tiene que estimar a partir de modelos matemáticos. También resalta la dificultad que representa el que la alfabetización se defina de distinta forma entre los países, así como la imposibilidad de catalogar la matrícula escolar por calidad de las escuelas.
} 
plia y los índices complementarios, que se discuten en el anexo de este documento, muestran que es posible hacer modificaciones para evaluar aspectos de interés específico (PNUD, 2003-1, pág. 15; López-Calva \& Vélez Grajales, 2003-1).

\section{Conclusión}

Dada la enorme recepción del concepto de desarrollo humano, la integración de este campo de estudio y acción se ha ido conformando más por agregación que orgánicamente a partir de un núcleo teórico único. Como consecuencia de este rápido y desfasado proceso el "nuevo" océano manifiesta dos características generales esenciales, aparentemente contradictorias: riqueza de investigación y reflexión teórica y dispersión de resultados y pluralidad de enfoques. Por una parte, el concepto desarrollo humano así como las metodologías y técnicas asociadas a él han sido una fuente prolífica de inspiración para promover investigaciones empíricas asociadas a las políticas públicas y acompañado diversas acciones colectivas, aunque, probablemente, han impactado en menor medida en los ámbitos estrictos de las universidades. Por otra, la dispersión y apresuramiento en su uso a menudo revelan vacíos teóricos y técnicos que ponen en duda su eficacia explicativa y pragmática.

La heterogeneidad teórica y metodológica más que un explicable y transitorio “desorden" conceptual y técnico, producto de un acelerado crecimiento de la actividad científica, tiene raíces más profundas afincadas en problemas teóricos históricos. Muchos de los logros y desaciertos se explican por los referentes teóricos y metodologías implícitos que conforman las investigaciones a veces implementadas sin crítica. Sobre todo si se considera que la profunda discusión colectiva que encabeza Sen se encuentra muy alejada por no decir inexistente en los trabajos empíricos.

Pero, esta amplia concepción del desarrollo no se queda en esta dimensión teórica. A partir del Informe de Desarrollo Humano del PNUD dos campos más de investigación se hacen evidentes. El primero, la presencia de una postura liderada por organismos internacionales que se traducen en políticas públicas nacionales e internacionales. Íntimamente ligado, al problema de la cuantificación del desarrollo y los esfuerzos técnicos empeñados en resolverlos.

En este amplio y difuso espectro tan relevante para la sociedad contemporánea el artículo presenta a la discusión, tanto teórica como técnica, la necesidad de hacer esfuerzos que ofrezcan esquemas operacionales que intenten integrar la perspectiva económica, sociológica política y filosófica para proponer aplicaciones del concepto en investigaciones puntuales pero también en la docencia. 


\section{Anexos}

\section{Anexo 1: El Índice de Desarrollo Humano (PNUD, 2003-6) (López- Calva L. F., 2003-6)}

El Índice de Desarrollo Humano surge como una forma de poner en práctica el desarrollo teórico de las capacidades de Sen. El índice se calcula y es publicado cada año en los Informes sobre Desarrollo Humano del PNUD. (PNUD, Estudios sobre el desarrollo humano, Num. 2006-6, 20036). La clasificación de cada país según el IDH se basa en tres medidas resumidas de funcionamiento: la esperanza de vida o el funcionamiento básico de vivir larga y saludablemente; el alfabetismo y la matriculación escolar o la habilidad de leer, escribir y adquirir conocimientos y finalmente, el ingreso (Producto Interno Bruto (PIB) per cápita ajustado por paridad y poder de compra (PPC)), que refleja el estándar económico de vivir o la habilidad para comprar los bienes y servicios que uno desea. La fórmula para calcular el IDH consiste en un promedio simple de las tres fuentes:

IDH $=1 / 3($ IEV) $+1 / 3($ IE) $+1 / 3$ (IPIB) (PNUD, Estudios sobre el desarrollo humano, Num. 2006-6, 2003-6)

En donde:

IEV = índice de esperanza de vida,

IE = índice de educación,

IPIB = índice de PIB.

Para el caso del Informe de Desarrollo Humano de México se adapta la fórmula original y genera un $I D H$ para cada uno de los Estados de la República, a partir del cual, se calcula el IDH nacional§§. La fórmula específica para obtener el IDH nacional es:

IEV $=\Sigma\{[$ IEVe - IEVmín m] / [IEVmax m - IEVmín m] $\}$ (PNUD, Estudios sobre el desarrollo humano, Num. 2006-6, 2003-6)

En donde el subíndice $e$ indica el estado de la República de referencia, el subíndice min $m$, se refiere al país del mundo que registra el menor valor de la variable en cuestión y el subíndice max $m$ al país con el mayor valor de la variable en cuestión.

El índice IE está, a su vez, compuesto de dos indicadores. En primer lugar, el índice de alfabetización de adultos (IA):

IA $=\Sigma\{[$ IAe - IAmín m] / [IAmax m - IAmín m] $\}$ y en segundo, el índice bruto de matriculación (IM): IM $=\Sigma\{[$ IMe - IMmín m] / [IMmax m - IMmín m] $\}$

en donde se aplican las siguientes ponderaciones: 


$$
\mathrm{IE}=2 / 3[\mathrm{IA}]+1 / 3[\mathrm{IM}]
$$

Por su parte, el índice del Producto Interno Bruto (PIB) por habitante, se calcula ajustando el PIB nacional por paridad y poder de compra (PPC) (con respecto al dólar de los Estados Unidos de América) para luego aplicar la fórmula: (PNUD, Estudios sobre el desarrollo humano, Num. 2006-6, 2003-6)

IPIB $=\Sigma\{[\log ($ PIBe $)-\log ($ PIBmín m) $] /[\log ($ PIBmax m) $]-$ $\log$ (PIBmín m)]\} Medición del Desarrollo Humano en México (PNUD, 2003-6): (PNUD, Estudios sobre el desarrollo humano, Num. 2006-6, 20036) (López-Calva L. F., 2003-6).

\section{Anexo 2: Bienestar basado en funcionamientos y capacidades de Sen (López-Calva L. F., 2003-6)}

Los bienes tienen un valor instrumental en tanto que permiten a los individuos un funcionamiento. Formalmente, sea $x i$ el vector de bienes de la persona $i$. Sen utiliza el hecho de que los bienes pueden ser convertidos en características. Por lo tanto, si $c$ es la función que convierte un vector de comodidades en uno de características, el vector de características consumidas por la persona $i$ estará dada por $c(x i)$. 㧊 $f i$ es la función (PNUD, personal de utilización que convierte características en funcionamientos. Dado que en este ejercicio $c$ es exógeno a la persona, podríamos pensar a $f i$ como una función que directamente convierte el vector de comodidades en funcionamientos.

En el modelo de Sen fi es parcialmente un asunto de la elección de la persona $i$. El individuo elige una función de utilización del conjunto posible, Fi, de las funciones de utilización. Un funcionamiento es una función que no dice que una persona $i$ ha alcanzado (como ser) dadas sus elecciones, una función de utilización fi __Fi. La representamos como:

$$
\text { bi }=f i(c(x i))
$$

El vector bi representa los resultados de utilizar las comodidades que se poseen y de elegir una función de utilización de Fi. Estos funcionamientos son por ejemplo, estar bien nutrido, bien vestido, tener un móvil y estar participando activamente en la vida de la comunidad. Desde el punto de vista aristotélico, esto implicaría funcionamientos en una forma humana (PNUD, Estudios sobre el desarrollo humano, Num. 2006-6, 2003-6).

Después, definiendo $\mathrm{Pi}(x i)$ como el conjunto de vectores de funcionamiento posibles para la persona $i$ como:

$$
\operatorname{Pi}(x i)=\left[b i \mid b i=f i(c(x i)) \text {, para algunos } f i \_F i\right] \text { suponiendo que la }
$$
persona $i$ tiene acceso a cualquier vector de bienes en $\mathrm{Xi}$. Entonces $\mathrm{Xi}$ 
representa sus derechos. Ahora, podemos definir la "libertad" efectiva que una persona tiene, dada su posesión de otros bienes y posibilidades individuales de convertir las características de los bienes en funcionamientos. Tal conjunto representa las capacidades de la persona $i$. Formalmente, la capacidad de la persona $i$ está dada por:

$$
\mathrm{Qi}=[\mathrm{bi} \mid \mathrm{bi}=\mathrm{fi}(\mathrm{c}(\mathrm{xi})) \text {, para algunos fi _ Fi , y para algunos xi _ Xi }] \text {. }
$$

Esto se suma a la formalización de Sen para ir desde los bienes, vía funcionamientos, a las capacidades. (PNUD, Estudios sobre el desarrollo humano, Num. 2006-6, 2003-6) (López-Calva L. F., 2003-6) 


\section{Bibliografía}

Ansa Eceiza, M. M. (2008). Economía y Felicidad: Acerca de la relación entre bienestar material y bienestar subjetivo. XI Jornadas de Economía crítica. Bilbao: Ecocri.

Basu, K. (1983). Cardinal Utility, Utilitarism and a Class of Invariance Axioms in Welfare Analysis. Journal of Mathematical Economics, Vol. 12

Bentham, J. (1789). An Introduction to the Principes of Morals and Legislation . Oxford: Oxford, Charenton, 1907.

Berlin, I. (1969). “Two Kinds of Liberty” . En I. Berlin, Four Essays on Liberty, Segunda Edición. Oxford: Oxford University Press.

Idem (1969). Four Essays on Liberty. Oxford: Oxford University Press.

Idem. (1969a). Two Concepts of Liberty. En I. Berlin, Four Essays on Liberty. Oxford: Oxford University Press.

Bruni, L., \& Porta, P. L. (2005). Economics and Happiness. Oxford.: Oxford University Press.

D’Apremont, C., \& Gevers, L. (1977). Equity and Informational Basis of Colletiv Choice. The Review of Economic Studies Vol. 4 .

Dobb, M. (1988[1975]). Teorías del valor y de la distribución desde Adam Smith. ideología y teoría económica. México: Siglo XXI.

Friedman, M., \& Friedman, R. (1990). Free to Choose. Harcourt: Harcourt. Griffin, K. (2001). “Desarrollo humano: origen, evolución e impacto”. En P. Ibarra, \& K. Unceta, Ensayos sobre desarrollo humano (págs. 25-42). Barcelona: Romanyà.

Ibarra, P., \& Unceta, K. (2001). Ensayos sobre el desarrollo humano. Barcelona: Rommaanayá Valls, s. a.

Lopéz-Calva, L. F. (2003). El concepto de desarrollo humano, su importancia y aplicación en México. En PNUD, Estudios sobre desarrollo humano Núm. 3. PNUD.

Idem (2003-6). Informe de desarrollo Humano. México: PNUD.

López-Calva, L. F., \& Vélez Grajales, R. (2003-1). Introducción: El concepto de desarrollo humano, su importancia y aplicación en México. En PNUD, Estudios sobre desarrollo humano PNUD México N. 3 (págs. 731). México: PNUD.

Maskin, E. (1978). A Theorem of Utilitarism. The Review of Economic 
Polis, Revista de la Universidad Bolivariana, Volumen 8, $N^{\circ}$ 23, 2009

Studies, Vol. 45 .

Morris, P. D. (1979). Measuring the Condition of the World's Poor: the physical quality of life index. Oxford: Pergamon Prees.

Nisbet, R. (1981). Historia de la idea de progreso. Barcelona: Gedisa.

PNUD (2003-1). Estudios sobre el desarrollo humano PNUD México No. 2003-1. México: PNUD.

Idem (2003-6). Estudios sobre el desarrollo humano, Num. 2006-6. México: PNUD.

Rawls, J. (1995[1993]). Liberalismo político. México: Fondo de Cultura Económica .

Rawls, J. (2006[1971]). Teoría de la justicia. México: Fondo de Cultura Económica.

Roberts, K. (1980). Interpersonal Comparability and Social ChoiceTheory. The Reviw of Economics Studies Vol. 47 .

Robinson, J. (s. f.). Carta de una economista Keynesiana a un economista marxista. México: s. e.

Salcedo M., D. (1994). Elección social y desigualdad económica. barcelona: Athropos.

Salcedo, D. (1998). La evaluación de las instituciones sociales según A. K. Sen. En A. K. Sen,

Sen, A. (1980). Equality of What? En S. M. McMurrin, The Tanner Lectures on Human Values (vol. I). Salt Lake City: Univ. of Utha Press.Sen, A. (1987a). On Ethics and Economics. California: Oxford: Blackwell.

Idem (1985). Commodities and Capabilities. Amsterdam: Amsterdam: North-Holand.

Idem (1999). Development as Freedom. N. Y.: Knopf Publishers. 